\title{
TCOM AMÉRICA LATINA \\ La identidad del campo de Comunicación de las Ciencias en América Latina
}

\section{Carina Cortassa}

\section{Resumen}

Palabras clave

DOI

\section{Introducción}

La Comunicación de las Ciencias en América Latina - en su doble dimensión de espacio de prácticas y de investigación — atraviesa en la actualidad un período de transición, de una etapa emergente a otra de consolidación. En este ensayo argumentaré que es preciso reforzar y acompañar ese proceso - caracterizado por el incremento de iniciativas, el interés de las políticas públicas e institucionales, la expansión del acervo de investigación empírica y el aumento de la masa crítica profundizando en una agenda de reflexión teórica y epistemológica con firme anclaje en el contexto regional.

Divulgación de la ciencia en los países en desarrollo; Comunicación científica: teoría y modelos

https://doi.org/10.22323/3.01010401

Fecha de recepción: 9 de julio de 2018

Fecha de aceptación: 27 de septiembre de 2018

Fecha de publicación: 21 de noviembre de 2018

\begin{abstract}
"Las ciencias nacientes - antes las psicológicas y sociológicas, ahora las comunicativas - son más ricas de intereses que de certezas. La pregunta por el estado actual es el reconocimiento de que todavía se está a la búsqueda de la identidad. Tiene sentido cuando permite reflexionar sobre los orígenes y no cuando cierra la interrogación con un balance de lo hecho." [Martín Serrano, 1990, p. 28]
\end{abstract}

El lanzamiento de JCOM - América Latina constituye un acontecimiento relevante para una comunidad académica y de prácticas que, mediante el esfuerzo y compromiso sostenidos, ha logrado durante la última década afirmar sus cimientos y proyección en la región. Este flamante espacio para el intercambio de perspectivas, investigaciones y experiencias en español y portugués — las lenguas 
que nos hermanan - puede considerarse, por una parte, el corolario natural de ese proceso de desarrollo; por otra, el punto de inflexión que señala una nueva etapa del campo, marcada por la posibilidad de diseñar una agenda temática más autónoma y anclada en las problemáticas que nos interpelan a diario. Una agenda que, sin desentenderse de las tendencias globales, encuentre en esta publicación la posibilidad de exponer los intereses e inquietudes de la ingente masa crítica que afronta cotidianamente al desafío de comunicar las ciencias, y de reflexionar sobre ello, en los países latinoamericanos.

Circunstancias como esta son especialmente favorables para el ejercicio de una mirada auto-reflexiva. Sin pretensiones de balance y clausura - como se señala en el epígrafe - sino más bien como un reconocimiento de las bases sobre las cuales estamos gestando, trabajosamente, la identidad de la comunicación y la cultura científica con una impronta regional particular.

Una serie de estudios recientes avalan la percepción de que el campo en América Latina atraviesa actualmente un período de transición de una etapa emergente a otra de consolidación y proyección. Al respecto, entre otros indicios cabe mencionar:

a. el creciente reconocimiento académico de la disciplina, la formación de masa crítica para la investigación y la profesionalización de recursos humanos, reflejada en la oferta de carreras de posgrado - maestrías, diplomaturas, especializaciones - y cursos de capacitación en algunos países [Massarani, Reynoso-Haynes y col., 2016];

b. el lento pero firme avance en las publicaciones de autores regionales y/o de artículos que abordan la problemática latinoamericana [Massarani, Rocha y col., 2017];

c. la estabilización de grupos locales y redes de investigación formales e informales (apoyadas por organismos y programas internacionales, como UNESCO, CYTED, OEI, entre otros);

d. la tendencia a la articulación y cooperación entre las comunidades de investigación y de prácticas, expresada por ejemplo en las contribuciones a los encuentros de especialistas; ${ }^{1}$

e. el progresivo interés de las políticas públicas regionales por la temática de la comunicación y la cultura científica [Polino y Cortassa, 2015;

Fernández Polcuch, Bello y Massarani, 2016].

Aunque abordar la evolución de un proceso en curso involucra riesgos - la falta de una perspectiva histórica más extensa puede resultar un obstáculo en este sentido - también representa un desafío estimulante. Bajo esa premisa, en este ensayo me propongo un doble objetivo. Por una parte, en la primera y la segunda

\footnotetext{
${ }^{1}$ Véanse, entre otras referencias, las memorias del $15^{\circ}$ Congreso de la RedPOP 2017 [RedPOP, 2018]; del Simposio de Comunicación Científica realizado en 2016 en Costa Rica [PCST, 2016]; de los sucesivos Congresos de Comunicación Pública de la Ciencia - COPUCI - que se llevan a cabo en Argentina desde 2011 [COPUCI, 2012; COPUCI, 2013] — y de los encuentros de la Sociedad Mexicana de Divulgación de la Ciencia y la Tecnología (SOMEDICYT), cuya vigesimosegunda edición tuvo lugar en 2018.
} 
sección planteo algunos interrogantes y dimensiones de análisis que podrían orientar ese examen en un plano general: ¿De qué hablamos cuando hablamos de la identidad del campo de Comunicación de las Ciencias en América Latina? ¿Cómo se articulan y se retroalimentan los espacios de prácticas y los espacios de investigación? ¿En qué medida las orientaciones adoptadas en ambos sentidos se corresponden con el nivel de desarrollo y las necesidades de la región y de los respectivos países? ¿Es posible - o aún, deseable - procurar establecer fronteras disciplinares que, sin renunciar a su hibridez constitutiva, reivindiquen la especificidad de los saberes teóricos, técnicos, prácticos, que estamos construyendo frente a los de otros campos cercanos?

El segundo objetivo es argumentar sobre la necesidad de que esos enfoques reflexivos estén más centrados en los contextos inmediatos de cada comunidad. Como afirmaré en la segunda y tercera sección, contribuir a la construcción de una cultura científica ciudadana más sólida y crítica es una de las metas que nos unifican disciplinarmente desde un punto de vista normativo. Pero qué significa una buena cultura científica en cada circunstancia no puede concebirse en un sentido homogéneo, pues eso se encuentra ligado a las condiciones del entorno en el cual se pretende desarrollarla. La comunicación de las ciencias orientada por ese mandato preceptivo debe entenderse como contextualmente dependiente: no puede ejercerse ni pensarse por fuera de las características y demandas de las realidades de los distintos países de la región. Es por eso que, más allá de los valores y fines últimos compartidos, sería positivo que cada comunidad encarase una agenda con acento en las particularidades locales y sus problemáticas concretas.

La Comunicación de las Ciencias: un campo plagado de solapamientos
Salvo por contadas excepciones [Rocha, Massarani y Pedersoli, 2017], la reflexión acerca de la construcción del campo de la Comunicación de las Ciencias en América Latina no constituye un aspecto demasiado abordado en la literatura o los encuentros de especialistas. ${ }^{2}$ Frente a la sistematización de experiencias y la exposición de resultados de estudios empíricos - temáticas habituales en las contribuciones a congresos y journals - las cuestiones vinculadas con las problemáticas epistemológicas de la disciplina, sus referentes conceptuales, la identidad de sus agentes respecto de ámbitos de conocimiento cercanos o afines, resultan comparativamente mucho menos frecuentes.

Esa situación tiene origen en factores de diverso orden. Un primer factor es de carácter temporal: aún podría aducirse - pero ya no por mucho tiempo — que, al tratarse de un área interdisciplinar y relativamente reciente, sus intereses se encuentran todavía más orientados al hacer, a la producción de un acervo - de hechos, de conocimientos — que a la especulación sobre sus fundamentos.

En segundo lugar, no es novedad que existe un solapamiento de intereses epistémicos y prácticos entre dos facetas del campo de Comunicación de las Ciencias que, en buena medida, añade complejidad al análisis. Esa duplicidad es reflejada en numerosas oportunidades por la literatura especializada en el modo de referirse al ámbito en cuestión: si en términos de un campo de estudios interdisciplinario y dinámico [Pardo y Calvo, 2002], como un área visible y reconocida de investigación académica [J. D. Miller, 1992], subrayando su

\footnotetext{
${ }^{2}$ Aunque el artículo está centrado en América Latina, sería interesante determinar, con evidencias fehacientes, si esa situación no es extensible a un plano más general.
} 
dimensión epistémica; o bien como el "movimiento" para la comprensión pública de la ciencia [Gregory y S. Miller, 1998], destacando su vertiente práctica, el sesgo militante o "misionario", como lo define Roqueplo [1983], de sus agentes. ${ }^{3}$

El tercer factor vinculado con la demanda auto-reflexiva que propongo ha sido señalado en numerosas oportunidades: se trata de la heterogeneidad de términos y expresiones empleados para aludir a las distintas dimensiones y niveles que se intersectan en el campo. Por ejemplo, analizando un conjunto de documentos de políticas públicas iberoamericanas sobre el tema, Polino y Cortassa [2015, p. 46] identificaron el uso indiscriminado de casi una decena de denominaciones: ${ }^{4}$ a) apropiación (social, pública, colectiva, de la ciencia, de la ciencia y la tecnología, del conocimiento científico); b) cultura científica; c) reconocimiento social de la CTI; d) visibilidad de la ciencia; e) alfabetización científica; f) percepción (de la ciencia, de la ciencia y la tecnología); g) divulgación, difusión, comunicación (social, pública, de la ciencia, de la ciencia y la tecnología, del conocimiento científico); h) popularización (de la ciencia, de la ciencia y la tecnología, de la ciencia, la tecnología y la innovación, del conocimiento); i) socialización y sensibilización del conocimiento. La misma falta de acuerdos es detectada tiempo después por Rocha, Massarani y Pedersoli [2017] al examinar la producción regional de artículos científicos sobre el tema.

Dejando de lado el elemento temporal, los aspectos de orden conceptual que exigen una revisión crítica se completan con un cuarto y último factor. Grosso modo desde comienzos de siglo, la influencia de los Estudios Sociales de la Ciencia y la Tecnología (CTS) trajo aparejada la apertura de los análisis de la comunicación y cultura científicas ${ }^{5}$ hacia nuevos objetos y procesos de investigación, al tiempo que propició la incorporación de enfoques que aportaron al campo sus respectivos intereses y marcos teóricos. A la perspectiva CTS se añadieron, progresivamente, contribuciones provenientes de la historia de la ciencia, la antropología cultural, la sociología y comunicación del riesgo, la psicología, la educación o la lingüística, entre muchas otras aproximaciones. Eso puede considerarse, por un lado, un avance positivo para un campo que, marcado en sus orígenes por el énfasis en los resultados empíricos, ${ }^{6}$ nunca había sido afecto a la sofisticación conceptual sino más bien débil en ese sentido. No obstante, también es necesario señalar que cuanto más se afirma el carácter interdisciplinar del estudio de la cultura científica, más lejana se percibe la viabilidad de establecer un umbral de acuerdos básicos, que permita unificar en alguna medida cuál es la especificidad del conocimiento que estamos produciendo. ${ }^{7}$

\footnotetext{
${ }^{3}$ Eso, sin agregar el problema que representa el acrónimo $C P C$ en lengua española, utilizado indistintamente para referir a los estudios de Comprensión Pública de la Ciencia (Public Understanding of Science) y Comunicación Pública de la Ciencia (Science Communication), cuyas diferencias, aunque sutiles, no deberían ser omitidas.

${ }^{4}$ Entre las cuales los autores proponen distinguir, mínimamente, aquellas que refieren a los fines de las prácticas $(a, b, c, d, e, f)$ y a los medios para alcanzarlos $(g, h, i)$.

${ }^{5}$ Shapin [1992, p. 29] cifra el peso de esa influencia en la obra de autores como Barnes, Bloor, Collins, Latour, Law, MacKenzie, Pickering, Pinch y Star.

${ }^{6} \mathrm{Me}$ refiero a las encuestas de percepción pública de la ciencia.

${ }^{7}$ Ese interés por un cierto grado de unificación no es necesariamente compartido. Para Locke [1999, p. 76], por ejemplo, la presencia de conflictos entre participantes de un campo no es motivo de preocupación sino para quien sostiene una visión idealizada de la investigación, en cualquier área de conocimiento, como internamente armónica y auto-consistente. No obstante, entiendo que el problema que señalo es previo a la existencia de conflictos: supone algunos acuerdos mínimos respecto de aquello sobre lo cual tiene interés entablarlos. Dicho de otro modo, mientras cada quien siga planteando los problemas de la comunicación de las ciencias en sus propios términos, es difícil que exista algo respecto de lo cual disputar.
} 
Disciplina

axiológica y

sociológica de la

in-disciplina

epistémica
Los solapamientos descritos en la sección anterior se traducen en inquietudes como las siguientes, expresadas en el Prólogo a las memorias de un congreso de la especialidad:

\begin{abstract}
¿Qué tienen en común un estudio sobre las concepciones de ciencia y tecnología de un grupo de estudiantes universitarios, los análisis de las noticias y las representaciones temáticas en la prensa y la televisión argentinas, y el examen de las relaciones entre estrategias de comunicación institucional y transferencia tecnológica? ¿Qué hilo conecta a la producción de contenidos didácticos para la enseñanza de la física nuclear con la elaboración de audiovisuales divulgativos y con la reflexión sobre unas jornadas de puertas abiertas a la comunidad? Dado que todas esas inquietudes aparecen reflejadas en las páginas subsiguientes, la pregunta que surge espontáneamente es cuál sería su común denominador." [Cortassa, Andrés y Wursten, 2017, p. 11]
\end{abstract}

En Cultural Boundaries of Science, Gieryn [1999] propone un método de "cartografiado cultural" para indagar en los procesos de configuración y reconfiguración de las disciplinas científicas, cuyas márgenes en constante movimiento resisten los criterios demarcatorios propios de las corrientes clásicas en filosofía de las ciencias Desde ese punto de vista, la ausencia de un núcleo problemático, teórico y metodológico homogéneo y plenamente compartido por los participantes - como describí en la sección anterior - no constituye un obstáculo insalvable para el reconocimiento de la identidad del campo de la Comunicación de las Ciencias. Esto es así porque, además de esos acuerdos fundamentales relativos a los modos válidos de conocer y teorizar acerca de la realidad, existen otra clase de factores - más allá de los de índole estrictamente epistémica - que permiten delimitar la especificidad de una comunidad de saberes y prácticas.

Frente a la cuestión inicial, entonces, una hipótesis plausible es pensar las fronteras disciplinares - y a partir de ello, las particularidades que adopta en América Latina - a partir de dos dimensiones: una, axiológica; la otra, institucional. Ambas subyacen a la disparidad terminológica, temática, conceptual y metodológica, dotando de coherencia y consistencia a la aparente fragmentación que predomina en el escenario.

Desde esa perspectiva, la Comunicación de las Ciencias puede concebirse como un campo de Investigación / Acción que - movilizado por valores políticos, sociales y éticos - integra diversos objetos, enfoques conceptuales e intereses epistémicos; intrínsecamente relacionados con el interés práctico de promover la construcción y expansión de una cultura científica pública, en cuyo marco los sujetos no solo logran integrar significativamente ciertos saberes a sus modos de conocer y representarse la realidad sino que también - a partir de ellos - se fortalecen en su condición de ciudadanos en un sistema democrático.

Lo que comparten sus agentes - académicos, practicantes - es un fin normativo y un conjunto de valores más que un núcleo de problemas, términos y teorías. Esa identificación como expertos en algo, aunque lábil, que les es común, es lo que sostiene el surgimiento y consolidación progresiva de una comunidad en sentido sociológico; esto es, dotada de estructuras institucionales para la incorporación y formación de nuevos integrantes, mecanismos de reconocimiento mutuo, ámbitos de encuentro avalados por la participación activa de sus miembros, y circuitos 
legitimados para el intercambio, la crítica y la construcción cooperativa de saberes. Es en ese marco de adhesión axiológica y sociológica adonde el grupo reconoce una especificidad que se impone frente a las fluctuaciones en las matrices epistémicas; el espacio de pertenencia delimitado por valores comunes que da contención, sin encorsetar, a la versatilidad intrínseca de sus inquietudes.

Cultura científica: una normatividad acotada
En la sección anterior sostuve que el valor atribuido a la promoción de la cultura científica ciudadana es un criterio normativo común a la diversidad de prácticas y enfoques englobados bajo el rótulo de Comunicación de las Ciencias. Considero que esa proposición es, en su generalidad, defendible. No obstante, es preciso señalar que la propia noción de "cultura científica" es polisémica. Eso conduce a una paradoja: habría un acuerdo generalizado acerca del valor de "algo" que debe promoverse - en un plano normativo - pero sin terminar de coincidir en qué es ese "algo" que se mantiene difuso desde un punto de vista descriptivo. Dicha ubicuidad es paralela a la falta de acuerdos firmes acerca de su significado y a su coexistencia ya señalada con otras categorías - comprensión pública de la ciencia, alfabetización científica o apropiación social del conocimiento. De allí también las disparidades en los mecanismos y prácticas destinados a su promoción en los diferentes países latinoamericanos - ¿cómo fomentarla? - y la determinación de sus fines - ¿por qué, para qué, hacerlo? - [Polino y Cortassa, 2015]. A esos interrogantes cabe añadir la pregunta por la cuestión evaluativa: ¿en qué consiste una buena cultura científica pública en cada contexto, y cómo determinarlo?

Distintas definiciones de cultura científica elaboradas durante las últimas dos décadas ponen el acento en sendas dimensiones del concepto [cfr. Godin y Gingras, 2000; Quintanilla, 1998; Quintanilla, 2010; Vogt, 2012]. Más allá de las preferencias subjetivas o grupales por una u otra, ${ }^{8}$ lo que interesa destacar es que todas esas aproximaciones, además de dar por sentado el valor que atribuyen a su consolidación, enfatizan el hecho de que la realización concreta de ese valor implícito no es generalizable sino contextualmente dependiente.

En su caracterización, Godin y Gingras [2000] distinguen tres modos o "canales" mediante los cuales los individuos y comunidades se apropian de la ciencia y la tecnología: a) el modo aprendizaje, a través del cual se adquieren y desarrollan los conocimientos, habilidades, representaciones, actitudes y valores necesarios para funcionar en un entorno permeado por ambas; b) el modo implicación, a través del cual la sociedad se beneficia de las habilidades de los individuos así formados para cumplir ciertas tareas vinculadas con ellas; y c) el modo socio-organizacional, a través del cual la sociedad crea y sostiene instituciones dedicadas a las actividades científicas y tecnológicas y a su control reflexivo. Si se admite la premisa de los autores de que "los individuos son sujetos sociales nacidos en un contexto social que les provee, mediante la experiencia, de una estructura inicial de socialización", entonces puede considerarse que el modo socio-organizacional, presumiblemente diferente en cada escenario, se encuentra en la base del proceso de apropiación social del conocimiento. Por ende, "[P]ara entender la cultura de los individuos, debemos por tanto entender la cultura de la sociedad circundante y sus diversas instituciones" [Godin y Gingras, 2000, p. 48].

\footnotetext{
${ }^{8}$ Marcadas por formación, trayectorias, adhesión a tradiciones, "colegios invisibles" o como prefiera llamársele.
} 
Según Quintanilla [1998] y Quintanilla [2010], el análisis de la cultura científica consiste, en una primera instancia, en la identificación de los componentes representacionales, prácticos y valorativos presentes en las dos dimensiones que la integran: el plano de la cultura intrínseca (conocimientos fácticos, de las reglas y hábitos que orientan sus prácticas de producción y de los valores - epistémicos, técnicos, sociales - que las guían) y el plano de la cultura extrínseca (imágenes públicas de la ciencia; códigos éticos, regulaciones políticas y jurídicas; valoraciones culturales, morales, políticas, religiosas). A esa etapa descriptiva, en la cual los componentes extrínsecos introducen las marcas distintivas de cada contexto, sobreviene otra evaluativa: se trata de examinar en qué medida las configuraciones detectadas son favorables al desarrollo general de la actividad científica y tecnológica, o por lo menos resultan compatibles con ella.

Finalmente, la metáfora visual de la "espiral de cultura científica" creada por Vogt [2012] $]^{9}$ refleja la interacción - incremental y retroalimentada — entre los espacios o cuadrantes de producción, reproducción y apropiación social del conocimiento científico. Pero el autor va más allá, señalando de qué manera esa dinámica se vincula necesariamente con la noción de orden más amplio de "bienestar cultural": tanto un concepto como un estado de espíritu, caracterizado por cierta sensación de confort social, crítico, frente a las inquietudes que generan los desafíos planteados por la tecnociencia [Vogt, 2012, p. 8], que pueden ser diferentes según adónde se los observe. Además de presentar un modelo analítico, el valor diferencial de la contribución de Vogt radica en su aplicación concreta a la realidad regional latinoamericana, y brasilera en particular.

¿Qué aportan esas referencias al hilo de mi argumento? A todas ellas subyace un presupuesto similar, implícito o explícito: el valor positivo que atribuyen a la construcción y expansión de la cultura científica. Al mismo tiempo, con sus respectivos matices, señalan que ese ideal normativo adoptará diferentes manifestaciones en función de una serie de condicionantes objetivos - sociales, políticos, culturales, educativos - propios del contexto en el cual se lo sitúe. Vista desde ese ángulo, queda claro por qué la reflexión sobre la identidad del campo de la Comunicación de las Ciencias en América Latina - en su doble dimensión de espacio de investigación y de prácticas - no puede disociarse del entorno en el que se inscribe. Son las circunstancias particulares de cada marco de referencia las que permiten distinguir, tanto en el nivel analítico como en el de intervención, cuáles son las acciones más apropiadas a los fines de lo que en cada escenario se postula como una buena cultura científica; esto es, aquella que tienda al empoderamiento de los ciudadanos y al fortalecimiento democrático. Ambas cosas no se logran de la misma manera en todos los casos, porque las necesidades y demandas a afrontar son diferentes.

En la región existen países en los cuales la alfabetización científica y tecnológica en el sentido más tradicional de la expresión - sigue siendo la materia pendiente que debe orientar el grueso de los esfuerzos, mal que pese a los detractores del modelo del déficit cognitivo. En otros casos, la ansiedad por implementar mecanismos de participación pública diseñados para sociedades con instituciones sólidas y transparentes, con sujetos familiarizados con la dinámica de las prácticas

\footnotetext{
${ }^{9}$ En cuya distinción entre los niveles "esotérico" (de producción y reproducción) y "exotérico" (de apropiación) de los hechos científicos y de su conocimiento relativo se advierten las huellas del planteamiento pionero elaborado por Fleck [1979].
} 
deliberativas informadas, ${ }^{10}$ ha derivado en ocasiones en resultados contraproducentes, frustrantes, precisamente por la enorme distancia en las condiciones objetivas de base. Finalmente, para no abundar, en muchos países celebramos la proliferación de acciones y recursos públicos para la comunicación de las ciencias, sin tener en cuenta que "más" no siempre es "mejor". Aunque en lo inmediato puede considerarse una señal de la consolidación del campo - tal como se indicó al comienzo de estas páginas - a mediano y largo plazo eso no constituye per se un indicador de evolución positiva. A diferencia de la energía, los recursos humanos y materiales aplicados sin una planificación y orientación precisas no se transforman: se pierden.

\section{Conclusión}

El estado actual de desarrollo del campo de Comunicación de las Ciencias en América Latina merece una reflexión epistemológica y teórica más acorde con los logros prácticos y de consolidación institucional alcanzados durante los últimos diez años. Además de un desafío intelectual apasionante, la tarea puede considerarse un elemento fundamental en la proyección disciplinar a futuro. Detenernos a pensar de dónde venimos, por qué caminos sinuosos hemos llegado hasta aquí, bajo qué condiciones comunes y específicas llevamos adelante nuestro trabajo practicantes e investigadores, qué necesidades y demandas imponen los respectivos contextos y qué compromisos adoptamos en relación con ellas, es un buen punto de partida para pensar hacia dónde deseamos dirigirnos en la incipiente madurez que asoma.

\section{Referencias}

COPUCI (2012). 'II Congreso de Comunicación Pública de la Ciencia (COPUCI)'. En: Número Especial Temático Congreso de Comunicación Pública de la Ciencia (COPUCI) 2012. "Fundamentos en Humanidades", año XIII, $n^{\circ}$ II (26) (San Luis, Argentina, 24-26 de octubre de 2012). Universidad Nacional de San Luis. San Luis, Argentina.

- (2013). 'III Congreso Internacional de Comunicación Pública de la Ciencia (COPUCI)'. En: Memorias (Rosario, Argentina, 11-13 de septiembre de 2013). Universidad Nacional de Rosario. Rosario, Argentina.

CORTASSA, C., ANDRÉS, G. y WURSTEN, A., eds. (2017). Comunicar la Ciencia: escenarios y prácticas. Memorias del COPUCI 2015. Paraná, Argentina: Universidad Nacional de Entre Ríos.

FERNÁNDEZ POLCUCH, E., BELLO, A. y MASSARANI, L. (2016). Políticas públicas e instrumentos para el desarrollo de la cultura científica en América Latina. Montevideo, Uruguay: LATU; UNESCO; RedPOP.

FLECK, L. (1979). Genesis and development of a scientific fact. $1^{\circ}$ ed. en alemán: (1935). Chicago, U.S.A.: University of Chicago Press.

GIERYN, T. F. (1999). Cultural Boundaries of Science: Credibility on the Line. Chicago, U.S.A.: University of Chicago Press. URL: https : //www . press . uchica go.edu/ucp/books/book/chicago/C/bo3642202.html.

GODIN, B. y GINGRAS, Y. (2000). 'What is scientific and technological culture and how is it measured? A multidimensional model'. Public Understanding of Science 9 (1), págs. 43-58. https://doi .org/10 . 1088/0963-6625/9/1/303.

\footnotetext{
${ }^{10}$ Me refiero, por caso, a experiencias que abrevan en las Conferencias de Consenso al estilo danés o formatos similares - foros, juicios o paneles ciudadanos.
} 
GREGORY, J. y MILLER, S. (1998). Science in public: communication, culture, and credibility. London, U.K. y New York, U.S.A.: Plenum.

LOCKE, S. (1999). 'Golem science and the public understanding of science: from deficit to dilemma'. Public Understanding of Science 8 (2), págs. 75-92. https://doi.org/10.1088/0963-6625/8/2/301.

MARTÍN SERRANO, M. (1990). 'La epistemología de la comunicación a los cuarenta años de su nacimiento'. Telos. Cuadernos de Comunicación, Tecnología y Sociedad 22, págs. 65-75. URL: http://eprints.ucm.es/13238/.

MASSARANI, L., REYNOSO-HAYNES, E., MURRIELLO, S. y CASTILLO, A. (2016). 'Posgrado en Comunicación de la Ciencia en América Latina: un mapa y algunas reflexiones'. [Spanish version of Science Communication Postgraduate Studies in Latin America: a map and some food for thought]. JCOM 15 (05), A03. https://doi.org/10.22323/2.15050203.

MASSARANI, L., ROCHA, M., PEDERSOLI, C., ALMEIDA, C., AMORIM, L., CAMBRE, M., NEPOTE, A. C., NOBERTO ROCHA, J., AGUIRRE, C., GONÇALVEZ, J. C., CORDIOLI, L. y FERREIRA, F., eds. (2017). Aproximaciones a la investigación en divulgación de la ciencia en América Latina a partir de sus artículos académicos. $10^{\mathrm{a}}$ ed. Rio de Janeiro, Brazil: Fiocruz, Casa de Oswaldo Cruz. URL: http://bit.1y/2vMYMgX.

MILLER, J. D. (1992). 'Toward a scientific understanding of the public understanding of science and technology'. Public Understanding of Science 1 (1), págs. 23-26. https://doi .org/10.1088/0963-6625/1/1/005.

PARDO, R. y CALVO, F. (2002). 'Attitudes toward science among the European public: a methodological analysis'. Public Understanding of Science 11 (2), págs. 155-195. https://doi .org/10.1088/0963-6625/11/2/305.

PCST (2016). Simposio Comunicación Científica como profesión: formación, responsabilidades y roles. Memorias. San José, Costa Rica: Vicerrectoría de Investigación, Universidad Estatal a Distancia.

POLINO, C. y CORTASSA, C. (2015). La promoción de la cultura científica. Un análisis de las políticas públicas en los países iberoamericanos. Papeles del Observatorio $\mathrm{N}^{\circ}$ 8. Buenos Aires, Argentina: Observatorio Iberoamericano de la Ciencia, la Tecnología y la Sociedad de la Organización de Estados Iberoamericanos (OCTS-OEI).

QUINTANILLA, M. A. (1998). 'Técnica y cultura'. Teorema XVII (3), págs. 49-69.

- (2010). 'La ciencia y la cultura científica'. ArtefaCToS 3 (1), págs. 31-48. URL: http://revistas.usal.es/index.php/artefactos/article/view/8428.

REDPOP (2018). ' $15^{\circ}$ Congreso de la RedPOP2017. Conexiones: nuevas maneras de popularizar la ciencia. (21-25 agosto 2017: Buenos Aires, Argentina)'. En: Memorias (Buenos Aires, Argentina, 21-25 de agosto de 2017). La Plata, Argentina: Universidad Nacional de La Plata.

ROCHA, M., MASSARANI, L. y PEDERSOLI, C. (2017). 'La divulgación de la ciencia en América Latina: términos, definiciones y campo académico'. En: Aproximaciones a la investigación en divulgación de la ciencia en América Latina a partir de sus artículos académicos. Ed. por MASSARANI, L., ROCHA, M., PEDERSOLI, C., ALMEIDA, C., AMORIM, L., CAMBRE, M., NEPOTE, A. C., NOBERTO ROCHA, J., AGUIRRE, C., GONÇALVEZ, J. C., CORDIOLI, L. y FERREIRA, F. $1 .^{\text {a }}$ ed. Rio de Janeiro, Brazil: Fiocruz, Casa de Oswaldo Cruz, págs. 39-58. URL: http://bit. 1y/2vMYMgX. 
ROQUEPLO, P. (1983). El reparto del saber. Barcelona, Spain: Gedisa.

SHAPIN, S. (1992). 'Why the public ought to understand science-in-the-making'.

Public Understanding of Science 1 (1), págs. 27-30.

https://doi.org/10.1088/0963-6625/1/1/006.

VOGT, C. (2012). 'The spiral of scientific culture and cultural well-being: Brazil and Ibero-America'. Public Understanding of Science 21 (1), págs. 4-16.

https://doi.org/10.1177/0963662511420410.

\section{Autor}

Cómo citar

Cortassa, C. (2018). ‘La identidad del campo de Comunicación de las Ciencias en América Latina'. JCOM - América Latina 01 (01), Y01.

https://doi.org/10.22323/3.01010401. 